\title{
UNA LEY PARA CONSOLIDAR LA REPÚBLICA: LA REFORMA ELECTORAL DE 1933
}

\author{
THE SPANISH ELECTORAL REFORM OF 1933. A LAW TO CONSOLIDATE \\ THE SECOND REPUBLIC
}

\author{
Roberto Villa García \\ Universidad Rey Juan Carlos, Madrid. España/Spain \\ roberto.villa@urjc.es
}

Recibido/Received: 09/04/2010

Aceptado/Accepted: 08/09/2010

\section{RESUMEN}

Este trabajo es un estudio de historia política sobre la ley electoral de 1933. Su importancia no puede ser soslayada, toda vez que la norma perfiló las reglas de juego a las que se amoldaron partidos y candidatos en los dos primeros comicios españoles, los de noviembre de 1933 y febrero de 1936, que fueron representativos de las innovaciones que introdujo la "política de masas" durante la etapa de entreguerras. La génesis y tramitación parlamentaria de esta reforma electoral, los intereses políticos que movían a sus patrocinadores y las consecuencias que tuvo en las estrategias y en los resultados electorales de los partidos en los dos comicios generales en los que esta ley estuvo vigente, son los principales objetos que aquí se analizan.

\section{PALABRAS CLAVE}

España, Historia política, Segunda República, elecciones, Ley electoral.

\section{SUMARIO}

1. Introducción. 2. La tramitación parlamentaria de la ley de 1933. 3. Hacia un consenso parcial con la oposición radical. 4. Los efectos de la ley electoral. Bibliografía.

\begin{abstract}
This work has the aim of being a monographic study of the electoral law of 1933. Its importance can not be ignored, since the standards outlined rules that parties and candidates adapted in the first two Spanish elections, the November 1933 and February 1936, which were representative of the innovations introduced by the "policy of mass" during the interwar period. The genesis and parliamentary process of this electoral reform, political interests which moved to its sponsors, and the consequences it had in the strategies and the electoral results of parties in the general elections in which this law was in force, are the main objects discussed here.
\end{abstract}

\section{KEYWORDS}

Spain, Political History, Second Republic, elections, Electoral Law. 


\section{CONTENTS}

1. Introduction. 2. The parliamentary proceedings of 1933 law. 3. Towards a partial consensus with Radical Party. 4. The effects of electoral law. References.

\section{INTRODUCCIÓN}

Los principios en los que se inspiraron las leyes electorales españolas han respondido, durante la larga trayectoria del Régimen Liberal en nuestro país, a las diversas teorías que sobre la representación se vinieron formulando desde principios de la centuria decimonónica. La cuestión pareció zanjarse en 1890 cuando todas las fuerzas políticas asumieron, con mayor o menor entusiasmo, el sufragio universal (claro que masculino) tras décadas de ampliación y reducción del cuerpo electoral al socaire de los considerandos, pero también de las supuestas conveniencias, de los distintos partidos. Al debate sobre el número de potenciales votantes, siempre se habían añadido otras cuestiones tales como las demarcaciones territoriales en los que éstos habrían de encuadrarse, la institución que confeccionaba el censo, y las garantías reales que la ley ofrecía para facilitar una concurrencia y competencia ordenadas, así como el fair play entre los distintos candidatos. Un análisis atento de los debates en torno a la normativa deja entrever que los posicionamientos de las diversas fuerzas políticas se ajustaban, prácticamente sin excepción, a una evaluación previa de los posibles beneficios o perjuicios que las reformas podían propiciar en sus expectativas electorales (Dardé, 2000 y 2003; Garrido, 2000; Estrada, 1999; Tusell, 1970; Tomás, 1968; Dérozier, 1965). Y esta evaluación, sin duda, partía de las experiencias previas que cada partido hubiera tenido en los comicios anteriores a la reforma. Que la confección de las leyes electorales se ajustara a intereses de partido no era algo ni propio sólo de España ni que haya de escandalizarnos hasta el punto de blandir esa afirmación en menoscabo del régimen liberal. Obviamente, a los partidos no les basta con tener votos para garantizarse una apropiada representación en el legislativo, sino que intentan (porque lo necesitan) maximizar esos sufragios para arramblar con el mayor número de escaños posible. El procedimiento electoral es el que permite la transformación de la voluntad del cuerpo electoral en actas de diputado, y de cómo la transmuta dependen no ya las posibilidades de los partidos de procurarse poder e influencia sino a veces su propia supervivencia.

Con todo, la historia de leyes electorales españolas nos permite observar que las modificaciones también respondieron a ansias, bastante objetivas, de regular $\mathrm{o}$ perfeccionar diversos aspectos del proceso electoral atendiendo a defectos previamente observados. La reglamentación cada vez más prolija de la constitución de la mesa electoral, de sus tareas durante las votaciones, del modo en que había de practicarse el escrutinio y la comunicación y publicidad de los resultados, el catálogo cada vez más amplio de delitos electorales y de sanciones, la creación de organismos independientes (las Juntas del Censo) encargados de dirigir y supervisar la elección, y la enumeración de las incapacidades e incompatibilidades en las que podían caer los candidatos, constituían los aspectos sobresalientes que más evolucionaron a lo largo del siglo XIX y principios del XX. Del mismo modo, aunque no pocas veces se impusieron los intereses de un sólo partido, el que en un momento determinado controlaba el ejecutivo o el legislativo (o 
ambos), a la hora de sacar adelante una ley electoral, en otras ocasiones ésta fue fruto de un relativo consenso. Es decir, en algunos momentos el partido "gubernamental" transigía con algunas de las vindicaciones de la oposición con el fin de que ésta no rompiese con el sistema político vigente y garantizase así la competencia electoral. Es necesario recordar que esta transigencia no significaba que el partido en el poder abjurase de sus intereses en beneficio de las formaciones opositoras, lo que hubiera supuesto el suicidio de su privilegiada posición política. Simplemente de lo que se trataba era de asegurar el pluralismo en el legislativo buscando, en cierto modo, extender (o al menos no disminuir) las bases de apoyo del sistema o, al menos, aminorar las oposiciones irreductibles.

En España, el número de leyes electorales "integradoras" no ha sido tan despreciable como, en un principio, pueda suponerse de la accidentada historia de nuestro régimen representativo. Dejando a un lado la reglamentación nacida de las Cortes de Cádiz, la primera ley relativamente consensuada fue la de 1837 en la que los progresistas aceptaron el principio del sufragio directo y ofrecieron una serie de garantías legales a la oposición moderada a cambio de que ésta accediese a una ampliación del cuerpo electoral en términos parecidos a la de la Reform Act británica de 1832. Integradora sería también la ley electoral de 1865, promovida por un gabinete de la Unión Liberal, que restableció las circunscripciones plurinominales y adoptó, incluso, demarcaciones electorales urbanas. Promovió también un nuevo aumento del número de electores y remozadas garantías legales para protestar y castigar el fraude y la corrupción, en un intento desesperado por sacar del retraimiento a los progresistas. Igualmente lo sería la de 1878, pactada entre los liberal-conservadores en el poder y las oposiciones "centralista" y "constitucional"; y la de 1890, en la que los conservadores acabaron aceptando el sufragio universal que propugnaban no ya los liberales sino los grupos antidinásticos. Por último, la ley de Maura de 1907 supuso una nueva ampliación del consenso no sólo a la oposición dinástica sino también a la antisistema que por entonces representaban los republicanos.

La legislación electoral en los años treinta de la centuria pasada no puede incluirse dentro de este grupo. Imitando lo sucedido en otras situaciones de brusca ruptura política (1836, 1854, 1868, 1873), el Gobierno Provisional de la República reformó por decreto la ley de 1907 . No se planteó comenzar un proceso de atracción de las fuerzas políticas que habían quedado fuera de la conjunción republicano-socialista afrontando la convocatoria de elecciones con la legislación anterior al 14 de abril de 1931, algo que sí había hecho Cánovas con la ley de 1869 (y la división en distritos de 1871) cara a los primeros comicios de la Restauración. A la espera de la ley electoral que habrían de confeccionar las nuevas Cortes constituyentes, el gabinete Alcalá-Zamora enmendó la norma de 1907 con dos disposiciones. La primera, de 25 de abril de 1931, variaba el método de formación de las listas de votantes entregando esta labor a unos Tribunales del Censo Electoral que habrían de ser designados para cada sección por las Juntas Municipales del Censo. Para esta labor habrían de requerir el auxilio obligatorio de dos funcionarios nombrados por los gobernadores civiles de entre los que cobrasen menos de cinco mil pesetas anuales. Además, todo el proceso habría de estar supervisado por el Ministerio de Trabajo, lo que suponía que los socialistas tendrían mayor ascendencia en una de las fases más importantes de la preparación administrativa de los comicios. Por último, la norma prejuzgaba una rebaja de la edad de voto al disponer que fueran censados todos los varones mayores de veintitrés años (Gaceta de Madrid -GM-, 26-IV-1931). 
La segunda disposición, la de 8 de mayo de 1931, supuso una corrección aún mayor de la legislación electoral vigente. El cambio más importante fue la supresión de todos los distritos uninominales y de la mayor parte de las circunscripciones plurinominales urbanas (exceptuando las de las once ciudades españolas que, en ese momento, sobrepasaban los cien mil habitantes), y su sustitución por demarcaciones de carácter provincial que elegirían un número de diputados proporcional a su población. La única excepción a la norma fue la creación de dos distritos uninominales: los de Ceuta y Melilla. En las sesenta y tres nuevas circunscripciones se establecía, además, un tope obligatorio del veinte por ciento de los votos emitidos para que un candidato pudiera ser proclamado en primera vuelta, habilitándose una segunda ronda en caso de que quedaran escaños por atribuir a causa de ese quorum. El voto limitado o restringido, que había imperado en las circunscripciones plurinominales de la Restauración, se extendía a todas las demarcaciones (excepto, obviamente, a las norteafricanas), de modo que el elector sólo podría votar un número de candidatos inferior a la cifra de escaños en juego ( $G M, 10-\mathrm{V}$ 1931).

En realidad, si el sufragio limitado parecía asegurar la presencia de la oposición reservándole un cupo de escaños, el tope del veinte por ciento y la segunda vuelta limitaban de consuno su eficacia. Si la segunda candidatura más apoyada no hubiera alcanzado al menos la quinta parte de los votos, los escaños de las minorías habrían de decidirse en una segunda ronda en que los partidos que hubieran triunfado, y se hubiesen llevado los diputados de las mayorías, podían volcar sus votos bien sobre candidatos propios sobrantes (proclamados, en principio, como auxiliares de los candidatos titulares), bien sobre candidatos de las formaciones políticas más afines. La exigencia de un tope y la segunda ronda electoral acentuaban el carácter mayoritario de una ley que, de por sí, ya lo era mucho más que la de 1907 al reducir drásticamente el número de circunscripciones electorales (recortando, por tanto, las demarcaciones por las que era posible presentarse) y al establecer cupos, tan desequilibrados como arbitrarios, para determinar cuántos escaños se llevaban los vencedores y cuántos los vencidos. Así, el cupo de las mayorías fluctuó en las distintas circunscripciones entre los dos tercios y los cuatro quintos de los asientos parlamentarios, constituyendo moneda común el atribuir a la candidatura vencedora tres cuartas partes de la representación independientemente del volumen de su victoria. Los restos (entre un quinto y un tercio de todos los escaños) se dejaban para la candidatura que se posicionaba en segundo lugar, mientras que las siguientes apenas si tenían posibilidades de obtener algún asiento.

La circunscripción provincial, la necesidad de traspasar un tope de votos y la enorme prima a la mayoría constituían incentivos diseñados por el Gobierno Provisional de la República para evitar el agrietamiento de la conjunción republicano-socialista que había triunfado parcialmente en los comicios municipales de 1931. Con un panorama político tan fragmentado como el español, ninguna fuerza política (ni tan siquiera el PSOE en los albores de la Segunda República) podía acreditar una implantación regular en casi ninguna de las nuevas demarcaciones electorales, y rara vez tan intensa como para agrupar por sí una fracción del electorado que sobrepasase en ese nuevo contexto territorial más de un veinte por ciento de todo el voto (Caro, 1997; Macarro, 2000: 36-50; Del Rey 2008: 98-99, 110-111, 147-148). Además, como hemos visto, la consecuencia de no triunfar era tan terrible (y el premio por la victoria tan apetitoso) que primaba la colaboración y la suma de fuerzas de los diferentes partidos de la conjunción que, en otras condiciones, ya se 
hubieran lanzado a competir electoralmente entre sí. De todas formas, si en una circunscripción concreta no se hubiera llegado a un acuerdo, la existencia de la segunda ronda remediaba el mal. Si las candidaturas rivales no llegaban al veinte por ciento del voto, republicanos y socialistas tendrían una nueva oportunidad para unir fuerzas y ganar los escaños.

Sin duda, el parcial remozamiento de la ley de 1907 tenía en cuenta cuáles eran los puntos fuertes de las fuerzas políticas monárquicas. La supresión del distrito uninominal estaba destinada a perjudicar a los viejos partidos de notables que se sustentaban sobre redes clientelares de carácter comarcal, tejidas además por liderazgos que raras veces sobrepasaban ese ámbito territorial. Esas redes ahora se revelaban como notoriamente insuficientes para alcanzar el escaño en las nuevas circunscripciones provinciales. Los republicanos y los socialistas estaban convencidos además, por lo sucedido en abril de 1931, que unas elecciones donde se movilizasen masas considerables de electores suponían, de por sí, una prima para ellos, pues habían demostrado saber hacerlo mejor que los monárquicos. Y por si acaso estos monárquicos quisieran reorganizarse haciendo valer el poder municipal conseguido el 12 de abril de 1931, desde los distintos gobiernos civiles se promovió, con la aquiescencia del Ministerio de la Gobernación, la poda de ayuntamientos más importante desde 1875-76. Según diversos estudios, unas dos mil quinientas corporaciones cambiaron de manos monárquicas a las de los diversos partidos republicanos y el socialista en tan solo mes y medio, teniendo este proceso colofón (que no comienzo) en los comicios locales parciales de 31 de mayo de 1931, a menos de un mes de las elecciones a Cortes constituyentes (Diario de Sesiones de las Cortes -DSC-, 2-VI-1933: Apéndice $2^{\circ}$ al no 349 ).

\section{LA TRAMITACIÓN PARLAMENTARIA DE LA LEY DE 1933}

La adopción de la circunscripción provincial, los topes y la segunda vuelta respondían, por tanto, a un interés político indudable. De todo esto dio fe la postrera reforma de 1933. En un contexto en que la conjunción republicano-socialista ya se hallaba cuarteada, y en el que la división de los partidos que sostenían el régimen había propiciado el triunfo de las derechas en no pocos de los ayuntamientos en disputa tras los comicios municipales de 23 de abril de 1933, el gobierno de Azaña presentó el 1 de junio de ese año un proyecto de ley que agudizaba más aún, si cabe, el sentido mayoritario del decreto de mayo de 1931 (DSC, 28-VI-1933: Apéndice $8^{\circ}$ al no 361). Es una lástima que las memorias y otros testimonios postreros apenas aborden el proceso de redacción de esta ley. En cuanto a la prensa coetánea, no hizo sino reproducir sobre el papel, y con parquedad, el debate que estaba teniendo lugar en las Cortes asumiendo la posición de los partidos afines.

En realidad, republicanos de izquierda y socialistas patrocinaban una disposición de un solo artículo que modificaba no la ley de 1907 sino el decreto de 8 de mayo de 1931 (que había sido convalidado por las Cortes constituyentes el 15 de octubre del mismo año). Los cambios afectaban, fundamentalmente, a los comicios municipales. No podía ser de otra manera dado que la experiencia electoral de abril de 1933 había resultado ejemplarizante y que los siguientes comicios de importancia, previstos para noviembre, habrían de renovar todas las corporaciones locales del país (aún cuando, como es sabido, 
ya nunca habría elecciones de este tipo en todo el periodo republicano, exceptuando las celebradas en enero de 1934 en las provincias catalanas). Los principales cambios, la supresión de la elección municipal por distrito y la ampliación a las grandes ciudades de la proporción que otorgaba a la lista vencedora entre tres cuartos y cuatro quintos de todos los puestos de concejal, suponían una agravación notable del principio mayoritario, que pretendía atemperarse con el establecimiento de una barrera como la del cuarenta por ciento para alcanzar las concejalías en primera vuelta. En líneas generales, este sistema consagraba sin rebozo la segunda vuelta y las candidaturas de coalición, así como una fortísima prima de escaños a la lista vencedora.

La agudización del sistema mayoritario de voto respecto a 1931 resultaba también perceptible en lo que concernía a las elecciones para diputados a Cortes. El número de ciudades con circunscripción propia se redujo al aumentar el guarismo necesario para constituirse como tal a 150.000 habitantes. Esto suponía, en la práctica, un descenso del número de circunscripciones totales que pasaban de sesenta y tres a sesenta. El requisito de que los candidatos triunfantes en primera vuelta obtuviesen como mínimo el cuarenta por ciento de los sufragios fue establecido de la misma forma que para los comicios municipales, doblando así la barrera del veinte por ciento establecida por el Gobierno Provisional. Para todo lo demás, seguirían rigiendo el decreto convalidado de 1931 y la ley electoral de 1907. El proyecto del gobierno pasó a la Comisión de Presidencia que, en lo que a las elecciones a Cortes se refiere, introdujo algunas modificaciones. Por de pronto, suprimió las circunscripciones de Ceuta y Melilla que pasarían a engrosar las de Cádiz y Málaga respectivamente. También modificó el porcentaje de votos necesario para obtener el escaño rebajándolo al treinta por ciento e introdujo un límite de sufragios por abajo, el doce por ciento, que habrían de superar los candidatos para pasar a segunda vuelta (DSC, 4-VII-1933: 13805).

En líneas generales, el dictamen de la Comisión no satisfizo no ya a las oposiciones sino a buena parte de los diputados gubernamentales. La lectura de éste tuvo lugar en la sesión del 4 de julio de 1933. Contra el dictamen se levantó, en primer lugar, un diputado de la ORGA, Ramón Suárez Picallo. Éste se mostró partidario de la representación proporcional y abjuró de las coaliciones electorales. El diputado coruñés rechazó la desmesurada prima a la mayoría afirmando que en casi todos los países democráticos con sistema mayoritario la proporción establecida era de dos tercios para la mayoría y el tercio restante para la minoría. Además denunció los efectos reales que con esa ley tendría la segunda vuelta electoral:

"Ponéis... esa auténtica trampa que significa la elección de segunda vuelta, ya que esa segunda vuelta representa que los que han ganado la elección en la primera vuelta irán al copo en la segunda... Yo no quiero suponer, Sres. Diputados, que este principio del proyecto de ley Electoral esté inspirado por el temor a que triunfen determinados sectores de la opinión española. Si esto es así, no es muy leal que digamos declararnos una República democrática".

Por último, a fuer de diputado de un grupo regional, abogó porque las mismas regiones constituyesen las circunscripciones electorales, petición que pretendía favorecer las expectativas de un partido como el de Suárez Picallo, movilizando a la opinión sobre la 
base de intereses y programas marcadamente regionalistas (DSC, 4-VII-1933: 1380413810).

Suárez Picallo había abogado por una ley electoral completamente nueva y por ello decidió no presentar enmiendas al proyecto. Esta postura sería también la que adoptase la minoría agraria, por boca de José María Gil-Robles. El líder de Acción Popular apuntó que el decreto de 1931 había mejorado la ley de 1907 al haber suprimido los distritos y establecido las circunscripciones provinciales. Pero resaltó que un sistema mayoritario como el que el Gobierno y la Comisión proponían sólo se aplicaba, en el siglo XX, en países dudosamente democráticos como Portugal e Italia. Gil-Robles destacó, además, la arbitrariedad del reparto de escaños entre mayorías y minorías:

“¿En virtud de qué una circunscripción que elige siete Diputados ha de tener cinco para la mayoría y dos para las minorías?... En el deseo exclusivo de favorecer el triunfo de las mayorías, y eso es, quizás, lo que haga simpático este proyecto a vosotros".

Se sumó, además, al coro de críticas contra los efectos perniciosos de la segunda vuelta que el diputado salmantino concretó en tres:

"En primer lugar, la segunda vuelta es una excitación a la violencia, porque conocido el resultado de la primera, todos los partidos agudizan toda clase de armas... para corregir, en una segunda vuelta, los posibles descalabros que hayan tenido... En segundo lugar, fatigáis innecesariamente al Cuerpo electoral, obligándole a movilizaciones continuas para una segunda vuelta... y en tercer término... colocáis en una posición de inferioridad a aquellos partidos políticos que cuenten con menos medios económicos”.

El proyecto de ley electoral traería consigo, según Gil-Robles, una aguda redimensión del mapa político y cambios bruscos en las mayorías parlamentarias:

"Este sistema... significa la muerte de los partidos intermedios... Los que se hallan en esta Cámara en una situación centro... estarán para siempre unidos a las fracciones extremas y no quedarán, en el choque de las pasiones políticas más que aquellos bandos separados irreconciliablemente, haciendo poco menos que imposible esas transacciones suaves, que son lo más eficaz en el orden político, por lo mismo que son las que eliminan las violencias... La prima a la mayoría, que... se puede volver contra vosotros, puede producir un movimiento de reacción tan violento como haya sido la acción salida de la obra revolucionaria, y no es ciertamente apetecible para un país que los movimientos de péndulo se produzcan de manera violenta... yendo a hacer tabla rasa del pasado para construir algo que el día de mañana puede ser destruido".

Frente al sistema que propugnaba la nueva reforma electoral, Gil-Robles defendió la representación proporcional aunque sin demasiadas esperanzas de convencer a la Comisión o a la mayoría parlamentaria para que retirasen el proyecto. Precisamente por ello, el diputado católico anunció la abstención de la minoría agraria en los debates que la 
ley habría de generar en esa y en las siguientes sesiones de Cortes. Aunque el jefe de la CEDA era coherente en la defensa de la representación proporcional, un principio que había defendido la primera formación política en la que había militado, el Partido Social Popular, no cabía duda que las derechas estaban especialmente alarmadas con la segunda vuelta y la prima a las mayorías, pues podían anular todo el esfuerzo de movilización en que habían empeñado el primer bienio si se formaba una sólida conjunción republicanosocialista contra ellas. En este sentido, la representación proporcional tenía la ventaja de conceder a los conservadores un número de escaños ajustado a su peso, creciente en la opinión pública a mediados de 1933 (DSC, 4-VII-1933: 13810-13813).

El tercer turno contra la totalidad, aunque desde un punto de vista más moderado, lo ejerció en nombre de su minoría el diputado radical Manuel Torres Campañá. A pesar de que su partido estaba dispuesto a presentar enmiendas y a colaborar con la Comisión, Torres afirmó al principio de su discurso que suscribía bastantes de las manifestaciones expuestas por Suárez Picallo y Gil-Robles. Este diputado arguyó contra la eficacia de la ley a la hora de constituir mayorías homogéneas que pudieran servir los intereses y la política de un Gobierno porque "los que lo han proyectado no conocen el mapa político español; el mapa político español no se presta a eso. Tal como están los partidos políticos... en España... eso no será posible en mucho tiempo". Enfiló también sus baterías argumentales contra la que consideraba exagerada prima para las mayorías: "el proyecto ha sido redactado... con un sentido de aplastamiento tal de las minorías, que no se concibe... que una mayoría de tipo democrático pueda presentar y sostener en estas Cortes la necesidad... de semejante proyecto de ley". Por último, acabó sumándose a las críticas de Suárez Picallo y Gil-Robles contra la segunda vuelta. El último turno corrió a cargo del diputado independiente Ossorio y Gallardo que insistió con dureza en los mismos defectos de la ley que habían señalado los oradores que le precedieron. En fin, el rechazo de las oposiciones al proyecto era tan frontal que la Comisión de Presidencia decidió retirarlo para su nuevo estudio (DSC, 4-VII-1933: 13813-13815).

No obstante, esa decisión había sido posible porque en esos momentos ejercía la portavocía de la Comisión un diputado radical, Pedro Armasa. Al día siguiente, el Gobierno y los miembros de la Comisión socialistas y republicanos de izquierda impusieron la discusión del dictamen sin rectificación. Este posicionamiento lo explanó un diputado del PSOE, Mariano Rojo. La intervención de Rojo resulta interesante porque permite conocer cuáles eran las consideraciones en las que se había fundado el proyecto:

"nosotros entendemos que para la mayor eficacia del régimen republicano, sobre todo en los momentos presentes... es preciso que puedan constituirse fuertes mayorías que permitan realizar una verdadera labor eficaz y que no sirvan, por el contrario, para estorbar la labor de los Gobiernos que se sienten en este banco azul".

Partiendo de que el gobierno consideraba que las formaciones republicanas representaban la mayoría de la opinión del país, éste pretendía forzar con una ley electoral extremadamente mayoritaria la coalición de los partidos que habían formado parte de la conjunción de 1931, y que dos años más tarde se encontraban cada vez más alejados entre sí. "No habrá más remedio", afirmó Rojo, “en un periodo más o menos largo, sino que 
haya una relación entre algunos sectores, los de más afinidad ideológica, para ir a estas elecciones" (DSC, 5-VII-1933: 13851).

Como el proyecto no fue retirado, comenzaron a discutirse las enmiendas. Un voto particular de los diputados radicales Armasa y Chacón pedía que las circunscripciones de Ceuta y Melilla continuaran como tal y no fuesen agregadas a las de Cádiz y Málaga respectivamente. En un principio, la mayoría de la Comisión rechazaba la enmienda, pero un discurso favorable de un diputado radical-socialista por Ceuta, Antonio López SánchezPrados, separó a su grupo de la mayoría gubernamental y provocó la derrota del ejecutivo por las oposiciones. No corrió tanta suerte otra de un correligionario de Sánchez-Prados, el diputado Navarro Vives, que pedía el restablecimiento de las circunscripciones urbanas en las ciudades que sobrepasasen los cien mil habitantes, tal y como lo contemplaba el decreto de 1931. Tampoco la tuvo otra del diputado radical Guerra del Río que pedía que se mantuviese la división de Canarias a efectos electorales establecida en 1912, y que respondía a pequeños distritos de carácter insular (DSC, 5-VII-1933: 13860-13868).

Los radicales pusieron, con otra enmienda de Torres Campañá, interés en reducir la prima a las mayorías e introducir algún tipo de criterio proporcional en la reforma electoral. Pero la Comisión, por boca del diputado socialista Heraclio Botana, se negó a cualquier transigencia. En cambio, no hubo problemas para que dos enmiendas del PSOE fuesen incorporadas: una que establecía que la propuesta de candidatos en los comicios municipales pudiera ser patrocinada por "entidades legalmente constituidas y que tengan su residencia en la localidad en que hayan de celebrarse elecciones municipales", concediendo tal facultad, en la práctica, a sindicatos y sociedades obreras; y otra que abría la posibilidad de que los candidatos a concejal pudieran ser presentados por un diputado o ex diputado (DSC, 5-VII-1933: 13868-13871).

La polémica se avivó cuando dos diputados socialistas, Mariano Rojo y José Ruiz del Toro, pidieron la supresión del requisito de que un candidato hubiera de sobrepasar el doce por ciento de los votos para poder acceder a la segunda vuelta, agravando así el sentido mayoritario de la ley y facilitando el copo de la representación por la lista más votada de una circunscripción. El diputado que defendió la enmienda, Ruiz del Toro, argumentó que la supresión de ese tope por abajo contribuía a acentuar el sentido democrático de la ley, pero la Comisión y el resto de partidos, que temían la prima que podía suponer para una formación con tanta fortaleza en 1933 como el PSOE, rechazaron tal posibilidad. No obstante, la negativa de los radicales y republicanos de izquierda tuvo carácter transaccional: estaban dispuestos a reducir el tope pero no a suprimirlo. Una propuesta del diputado radical-socialista José Salmerón, que lo rebajaba del doce al ocho por ciento, fue finalmente admitida por los socialistas (DSC, 6-VII-1933: 13892-13894).

\section{HACIA UN CONSENSO PARCIAL CON LA OPOSICIÓN RADICAL}

La discusión se puso al rojo vivo con una enmienda del progresista Castrillo Santos que pretendía, sencillamente, abolir cualquier tipo de requisito de topes mínimos y máximos, y restablecer el principio, ya tradicional en las leyes electorales españolas, de que los candidatos con mayor número de votos escrutados fueran automáticamente electos diputados, sin necesidad de recurrir a ningún tipo de segunda vuelta. La enmienda nacía muerta por la anterior transacción entre la mayoría y el Partido Radical, pero Castrillo 
aprovechó su intervención para lanzar una nueva carga de profundidad contra el dictamen. Probablemente estaba defendiendo el punto de vista del propio Alcalá-Zamora que había rechazado de plano un proyecto de reforma electoral más marcadamente mayoritario que el primitivo dictamen y al que tampoco gustaba la excesiva prima a las mayorías que concedía el proyecto del gobierno (Alcalá-Zamora, 1977: 243). Este testimonio concuerda con el de Azaña, que testimonia la angustiada petición del jefe del Estado para que, al menos, se elevase todo lo posible el porcentaje mínimo de votantes para alcanzar la victoria electoral en primera vuelta (Azaña, 1997: 388).

Castrillo volvió contra las razones expuestas al principio del debate, tachando la ley de estar inspirada en designios fascistas aún más agravados, ya que al menos con la ley de Mussolini se reservaba a las oposiciones una cuarta parte de la representación parlamentaria, mientras que con la reforma electoral de Azaña:

“...nos encontramos con esa cosa enorme que se llama segunda vuelta, que consiste en el derecho que tienen las mayorías de volver a votar, aniquilando prácticamente a las minorías... Yo pregunto... ¿Hay alguna razón de conveniencia táctica... hay alguna razón de justicia para ofrecer a nuestros conciudadanos el ejemplo de esta ley brutal... que no responde a ninguno de los sistemas conocidos en el mundo?".

El orador se negaba incluso a aceptar que las modificaciones en la Cámara significasen mejora alguna en la ley, sobre todo respecto al decreto de mayo de 1931. Y era que Castrillo y los firmantes de la enmienda, pertenecientes a pequeños o medianos partidos como el progresista, la Lliga o la Izquierda Radical-Socialista, temían quedar laminados electoralmente al sobredimensionarse la representación de los grandes partidos (DSC, 6-VII-1933: 13896).

La Comisión se negó a admitir su enmienda, pero Castrillo había logrado el propósito de levantar a las oposiciones de nuevo contra la totalidad del dictamen. Botella Asensi, diputado disidente del radical-socialismo, consumió un turno de palabra para apoyar los argumentos del grupo progresista. El portavoz de la minoría radical, Guerra del Río, también habló en contra del dictamen y a favor de la enmienda de Castrillo. Se quejaba Guerra, además, de que la mayor parte de las enmiendas del Partido Radical habían sido rechazadas por la mayoría. Tras la impugnación que hizo del proyecto el comunista Balbontín, se levantó el portavoz de la Lliga, Pedro Rahola. Éste observó que la ley en discusión obedecía no a criterios objetivos sino a uno puramente interesado y oportunista:

"Si se es partidario del sistema mayoritario, señores del Gobierno, señores de la Comisión, se ha de ser partidario del sistema mayoritario... con todas sus consecuencias, y entonces, si venís hablando de un tope, este tope no puede ser más que uno, que es más del 50 por 100 de todos los votantes... Pero el tope del 40 , del 30, del 20, del 10, reconoceréis que es un tope absolutamente arbitrario, que no obedece más que a la comprensión, después de examinar el estado de los distritos o provincias, de las posibilidades que ofrece ocasionalmente la elección para triunfar unos u otros partidos: es decir, un concepto... de pura conveniencia". 
El diputado catalanista acabó su intervención calificando el sistema que consagraba el dictamen como "un fascismo vergonzante, porque tiene por objeto asegurar el predominio de un partido o de un grupo de partidos, desconociendo en absoluto cantidades enormes de opinión que tienen derecho a ser oídas y respetadas". A Rahola le siguió el portavoz del PNV, Leizaola, y del grupo agrario, Royo-Villanova, que, insistiendo en las mismas motivaciones, anunciaron su apoyo a la enmienda de la minoría progresista. El diputado Ossorio Gallardo mostró también su conformidad con Castrillo, lamentó que la Comisión insistiese en presentar el dictamen tal y como estaba redactado, y lo achacó al gobierno, sobre todo al propio Azaña, al que pidió que interviniese en el debate para aclarar sus motivaciones (DSC, 6-VII-1933: 13896-13904).

El presidente del Consejo intentó conjurar el vendaval con un larguísimo discurso en el que dejó entrever las intenciones del gabinete, motivaciones que no se separaban de lo argumentado por los miembros de la mayoría en la Comisión. Destacó, en primer lugar, que el decreto de 1931 sólo se promulgó para los comicios constituyentes y que, por tanto, resultaba necesario habilitar un cauce legal para poder hacer frente a una convocatoria electoral inmediata. Descubrió que la reforma electoral partía del supuesto de sus autores "de que los republicanos de todos los colores y los socialistas juntos tenemos la inmensa mayoría del país". No obstante, en el mismo discurso, Azaña rechazaba que la redacción hubiera tenido algún propósito partidista. Cuando entró a analizar la ley, éste preguntó a las oposiciones dónde estaba el posible "aplastamiento de las minorías" en el proyecto, y justificó la segunda vuelta de esta guisa:

"Votación en primera vuelta, en única vuelta; supongamos el caso: dispersión de las candidaturas republicanas en diversos grupos de votantes y triunfo de una candidatura con menor número de votos que todas las demás candidaturas juntas. ¿Qué pasa entonces?... Entonces, ¿no se han dejado perecer el derecho de los representantes y votantes a favor de una minoría?... Porque hay que atender, en efecto, a que no se proscriba el derecho de las minorías; pero hay que evitar este otro peligro... que es la posible dispersión de las candidaturas republicanas y socialistas, faltas de coalición, y su derrota por una candidatura antirrepublicana y antisocialista, minoritaria, que represente mucha menos fuerza que todas las demás candidaturas republicanas y socialistas... si se hubieran sumado en una coalición".

Por tanto, la reforma electoral tendía a conservar la República a través de la misma salvaguardia de la conjunción republicano-socialista:

"Si nosotros no tuviéramos la mayoría del país, no habría ni cuestión, ni habría que hablar, y todo el tinglado electoral estaría de más; pero teniendo la mayoría en el país, hay que dar los medios legales, normales, jurídicos... para que los efectos políticos de la disciplina de los partidos se produzcan normalmente, y para que los efectos de la disgregación o de la indisciplina de los partidos o de la mala inteligencia entre los partidos, no causen a la República... daños irreparables. Sería altamente extraño que por no prever este caso, minorías monárquicas o antirrepublicanas trajeran a la Cámara una representación que no les correspondiera". 
Y, sin embargo, cerró su discurso reafirmando el carácter neutro de la ley: “a nadie se le ha pasado por las mientes, señores Diputados, inventar un sistema electoral para asegurar la permanencia en el poder de una coalición de partidos" (DSC, 6-VII-1933: 13904-13911).

La oración no convenció en absoluto a las oposiciones pero tuvo un efecto positivo en los partidos de la mayoría, que cerraron filas en torno al gobierno. Los oradores de las minorías no encontraron una grieta por donde forzar la retirada del dictamen. Ossorio Gallardo insistió en que la ley en proyecto suponía el aplastamiento de las minorías. Al argumento de Azaña de que había que incentivar a los partidos republicanos para que se coaligasen en bien del régimen, respondió con relativa sencillez:

"S. S... ahora dice: 'Tenemos que inventar un artificio para que donde se desunan y escindan los partidos republicanos, no pueda introducirse su adversario'. Y yo le digo: 'señor Azaña, eso no es leal; si llega ese caso, no invente S. S. artificio para evitarlo, porque será que la vida española en aquel sitio ha dado eso de sí y no será lícito falsificarlo con una prevención y con unos arbitrios como los que esta ley contiene" (DSC, 6-VII-1933: 13913).

La firmeza de las oposiciones contra el dictamen y, sobre todo, el alineamiento del Partido Radical con éstas, determinó que la Comisión de Presidencia, a instancias del gobierno, anunciara la introducción de algunas modificaciones, pero sin retirar el proyecto. Éstas se publicaron en el Diario de Sesiones el 13 de julio. Consistían, fundamentalmente, en que la proporción de escaños entre mayorías y minorías se establecería en dos tercios para las primeras y un tercio para las segundas, pero sólo en las elecciones municipales. En caso de que la división en tercios no fuese exacta y quedaran restos, estos pasarían a formar parte del cupo de las mayorías, con lo que en realidad apenas se aminoraban las proporciones iniciales. También variaban los porcentajes mínimos de voto escrutado para obtener el escaño. Se evitaría la segunda vuelta siempre que al menos un candidato hubiera sobrepasado el cuarenta por ciento de los sufragios emitidos y que todos los demás hubieran obtenido como mínimo el veinte por ciento de los sufragios. Si ningún candidato hubiera llegado al cuarenta por ciento de los votos, habría de ser necesaria una segunda vuelta en la que se dirimirían todos los escaños. En el caso de que esto sí sucediese, pero que algún escaño no se hubiera provisto por candidatos que reuniesen al menos un veinte por ciento de los votos, la segunda vuelta sólo tendría lugar para cubrir estas vacantes. A esta segunda vuelta sólo podrían concurrir, como en el debate del 6 de julio se había establecido, los candidatos que hubieran reunido como mínimo un ocho por ciento de los sufragios emitidos (DSC, 18-VII-1933: Apéndice $3^{\circ}$ al no 372).

La nueva redacción suponía un intento de combinar el criterio agudamente mayoritario sostenido por el gobierno con una nueva bajada del listón, del treinta al veinte por ciento, que permitiese encontrar algún punto de encuentro con la oposición. No obstante, no tuvo el asentimiento de toda la Comisión. Tres miembros del Partido Radical presentaron un voto particular rebajando aún más los topes mínimos. Pero esta postura resultaba inaceptable para el gobierno, pues hubiera supuesto reducir tanto los requisitos que hacía por completo innecesaria la repetición de las elecciones y, por tanto, hubiera 
desincentivado algo más las coaliciones. En este apartado, el más importante, la reforma quedó fijada y ya por completo invariable (DSC, 19-VII-1933: 14223-14227, 14231).

El resto de modificaciones careció del mismo grado de polémica. El diputado radical Torres Campañá pudo retocar un tanto el dictamen gracias a que la Comisión incluyó dos de sus enmiendas. La primera, para el caso de que ningún diputado obtuviese el ocho por ciento de los sufragios y quedasen vacantes por cubrir, que la elección fuese declarada libre, pudiendo concurrir en segunda vuelta todos los candidatos independientemente de la cifra de votos obtenida en la primera. La segunda enmienda necesitó de mayor discusión. Pedía Torres que, en caso de que sólo existiese un número de candidatos similar al de puestos a cubrir que hubieran logrado sobrepasar el límite del ocho por ciento pero no el del veinte por ciento, fuesen electos diputados automáticamente. La medida parecía necesaria, toda vez que no tenía mucho sentido convocar una segunda vuelta en la que hubiese tantos candidatos como puestos vacantes. Este aserto convenció a la Comisión que acabó aceptando la enmienda. Una postrera intervención de Ossorio pidiendo, infructuosamente, que se estableciera la fórmula del voto acumulativo que había estado vigente entre 1878 y 1890 cerró definitivamente el debate sobre la reforma electoral (DSC, 19-VII-1933: 14232-14236 y 14241-12242), y ésta fue aprobada al día siguiente, 20 de julio de 1933.

\section{LOS EFECTOS DE LA LEY ELECTORAL}

En líneas generales y pese a las críticas, la definitiva redacción de la norma mejoraba algo no sólo el primitivo proyecto del gobierno sino, hasta cierto punto, el decreto de 1931. De hecho, teniendo en cuenta el ánimo exclusivista que lo había inspirado al principio, el proyecto de ley acabó modificándose gracias al interés del ejecutivo en sumar el apoyo de los radicales. Aunque éstos ya se habían declarado en franca oposición al gabinete Azaña, el partido de Lerroux era un importante factor a tener en cuenta para las próximas elecciones locales y las siguientes generales (como habían mostrado los resultados de los comicios municipales parciales de abril de 1933). Cierto que Azaña y otros dirigentes republicanos de izquierda demostraron que sus preferencias se orientaban hacia una alianza electoral con el PSOE, pero en los lugares donde la oposición conservadora mostrase mayor arraigo y se llegara a una segunda ronda, parecía conveniente tender puentes hacia los radicales cara a establecer coaliciones circunstanciales. De ahí que, a pesar de que en un primer momento se desecharon la mayor parte de las enmiendas del PRR, cuando a partir de la intervención de Castrillo el gobierno pudo avistar el alineamiento de esta minoría con el resto de las oposiciones, frenaron la tentativa de sacar adelante el primitivo proyecto de ley y atisbaron algún tipo de entendimiento con ella.

Ese relativo consenso limó algunas de las aristas del decreto emitido hacía más de dos años por el Gobierno Provisional. Si en 1931 bastaba con que la lista vencedora sobrepasase simplemente el veinte por ciento para obtener entre un 67 y un 80 por ciento de los escaños de una circunscripción, ahora por lo menos se le exigía que uno de sus candidatos llegase a sobrepasar el cuarenta por ciento de los sufragios emitidos. Además, las opciones minoritarias quedaban mejor protegidas al exigirse a los candidatos obtener un porcentaje mínimo, el tope del ocho por ciento, para poder pasar a la segunda vuelta. Con el decreto de 1931 resultaba posible la presentación de candidatos secundarios, o hasta 
candidatos nuevos, de un mismo partido en la segunda ronda con el fin de que, tras haber sacado triunfante la lista por las mayorías, se pudiera obtener el copo de la representación. La elección automática en primera vuelta de un número de candidatos similar al de vacantes, aunque no cumplieran con el tope del veinte por ciento pero siempre que obtuviesen el mínimo del ocho por ciento de los sufragios emitidos, representaba también otra pequeña garantía para las minorías que no existía en 1931.

No obstante, aunque la ley de 1933 no suponía, a causa del voto restringido, el retorno puro y simple al viejo régimen electoral de los primeros tiempos del liberalismo español, sí que la adopción definitiva de la circunscripción provincial representaba un retorno al pasado, así como una agudización de los efectos más nocivos del sistema mayoritario, incluso respecto a 1907 (Gunther, Sani y Shabad, 1986: 21). En este sentido, los republicanos amalgamaban su preferencia por la tradición francesa de los distritos plurinominales basada en las circunscripciones departamentales (Campbell, 1958, Rémond, 1990; Huard, 1997), con su también clásica predilección por el modelo de elección que se venía realizando en las circunscripciones urbanas españolas desde hacía varias décadas. Por ello, de 1931 en adelante, no hicieron más que trasplantar al mundo rural las reglas electorales que ya se practicaban en las ciudades: el plurinominalismo, el voto restringido y el cupo a las minorías, presumiblemente a modo de receta para procurar el "despertar" de la opinión en los pueblos.

La reforma de 1933 traía consigo, además, algunos riesgos. La reducción del número de circunscripciones, aunque resultara tan modesta (sólo tres), contribuía desde el punto de vista territorial a agravar la prima a la mayoría. Hemos de tener en cuenta que, cuantos más puestos de diputado poseía una circunscripción, más se desvirtuaba la relación de escaños entre mayoría y minoría, mientras que cuanto menos, la distribución de los escaños entre ambos grupos resultaba relativamente más proporcionada. Por ejemplo, en la circunscripción de Soria, que poseía tres escaños, la diferencia entre obtener las mayorías (dos) y obtener las minorías (uno) suponía tan sólo un escaño; en Álava, que tenía dos, no había diferencia alguna; en Guadalajara, que tenía cuatro, sólo dos; en Castellón, que tenía seis, sólo dos; en Salamanca que tenía siete, ya eran tres. Mientras tanto, el triunfo por un solo voto en las circunscripciones urbanas de Madrid o Barcelona representaba la friolera de nueve escaños de diferencia, lo mismo que en Oviedo o La Coruña.

En definitiva, cuantas más circunscripciones, mejor repartidos estaban los escaños y mayores posibilidades tenían los partidos de lograr una minoría respetable haciéndose fuertes en varios distritos. Por ello, en la práctica, un sistema tan aparentemente agravado como el de 1907, en el que exceptuando las circunscripciones urbanas sólo se podía obtener representación ganando en algún distrito, facilitaba paradójicamente el acceso a las Cortes de las minorías, pues una pequeña fuerza política podía concentrar su labor en unos pocos distritos y, dado su elevado número, las oportunidades de conseguir representación eran mucho mayores. Con el sistema de 1931 y de 1933, un partido necesitaba posicionarse como primera o segunda fuerza política en toda una provincia para obtener representación. En caso contrario, estaba prácticamente obligado a coaligarse con otros partidos. Una de las consecuencias previsibles de ese sistema electoral, en tanto que actuaba sobre un sistema de partidos apenas recién nacido, podía ser el de dificultar la formación de candidaturas, en la medida en que sin partidos nacionales fuertes y bien implantados, las coaliciones se hacían casi inevitables, en medio de una marcada y casi ineluctable tendencia a la bipolarización. Porque si dos fuerzas políticas secundarias se 
unían acrecentando de ese modo sus posibilidades de victoria sobre el partido con más arraigo en la circunscripción, éste se veía empujado a acudir a la coalición con otra más pequeña para volver a restaurar su primacía, y así hasta el infinito. El resultado previsible era, dada la necesidad de alcanzar la barrera del cuarenta por ciento, la presencia de dos grandes coaliciones que habrían de luchar entre ellas a brazo partido por una victoria a la que incitaba la desbordante prima a las mayorías. Con la ley de 1907, la derrota en un distrito no suponía para un partido más que un pequeño descalabro que podía ser compensado con la victoria en otro de los varios cientos existentes. Sin embargo, con el sistema de 1933, la derrota en una o dos provincias clave, aunque fuese por una diferencia mínima de votos y dada la drástica reducción de circunscripciones, podía resultar toda una catástrofe, ni más ni menos que la derrota electoral misma. Además, la reforma de 1933, en un hipotético contexto en que se hubiesen evitado amplias coaliciones, facilitaba que un partido con relativa fortaleza como para posicionarse en primer lugar, pero con ciertas discontinuidades en su implantación territorial (pues podía concentrar sus bases de apoyo, por ejemplo, en la capital de provincia y en un número reducido de comarcas) pudiera acarrear con la mayoría abrumadora de la representación provincial. Mientras, con la ley de 1907, este partido necesitaba una implantación en todos y cada uno de los distritos comarcales para obtener una victoria de semejantes proporciones, algo que resultaba bastante más complicado y que por tanto aseguraba la presencia de otras fuerzas políticas del mismo territorio.

Del mismo modo que los cambios en el reglamento del Congreso (Álvarez Tardío, 2003: 203-205), la reforma de 1933 supuso un apreciable espaldarazo para las grandes organizaciones partidistas, de programas con fuerte contenido ideológico, extendidas a nivel nacional o en un número de provincias determinado, frente al predominio de la política personalista, pragmática y menos ideologizada que caracterizó a la Restauración. La aspiración primaria de un partido residía en obtener por sí mismo la fuerza suficiente en una circunscripción como para derrotar a una entente de partidos rivales y conseguir la valiosa prima a las mayorías. Pero como esto resultaba bastante difícil en un sistema de partidos tan fragmentado como el español, que acababa de nacer de la forma más improvisada e inesperada posible, la pretensión más importante e inmediata era nuclear una coalición en torno suyo (y obtener la primacía a la hora del reparto de puestos) o, al menos, hacerse imprescindible a la hora de constituir candidaturas conjuntas e impedir así la tragedia de quedar aislado. De hecho, la existencia de la segunda vuelta, motivada por la no superación del cuarenta por ciento de los sufragios emitidos en ese contexto de marcada fragmentación, estimulaba hasta tal punto las coaliciones que el objetivo de muchos partidos pequeños se cifró, más que en conseguir la victoria electoral, en sacar un número de sufragios suficiente en la primera vuelta como para exigir en las mejores condiciones posibles puestos en una futura candidatura de coalición cara a la segunda vuelta. Evidentemente, detrás de todo ello había un análisis, hasta un cálculo si se quiere, muy claro del arraigo de cada partido político. En el estío de 1933 se consideraba, con no poca razón, que muy pocas formaciones tenían la fuerza suficiente como para hacerse, en solitario, con las mayorías en buena parte de las provincias españolas.

Dada la corta experiencia republicana, resulta de todo punto imposible suponer que este régimen electoral pudo haber favorecido la creación de dos grandes partidos. Resulta incuestionablemente cierto que entre 1932 y 1936 estaba en marcha un proceso de cristalización de un sistema de partidos, que apenas si había estado bosquejado en las 
elecciones a Cortes Constituyentes (Linz, 1979: 171-180; Juliá, 1995: 138-139). Ahora bien, existen muy pocas evidencias de que se caminase decididamente hacia la desaparición del faccionalismo o de la fragmentación. Más bien todo lo contrario. El proceso en marcha parecía conducir a un sistema de dos grandes coaliciones multipartidistas, en las que el fenómeno del faccionalismo seguiría primando sobre los principios de cohesión y disciplina (Varela, 1978: 59-60; De Carreras, 1983: 165). Con un panorama político tan dividido, que se caracterizaba por la abundancia de opciones ideológicas organizadas, la necesidad de coaliciones, de sumar fuerzas políticas aún pequeñas para obtener el máximo posible de votos, hizo posible la subsistencia de las viejas maquinarias caciquiles manejadas por individuos con tradicional arraigo en determinadas comarcas de una provincia que, de este modo, lograron aún hacerse un hueco en las candidaturas. En este sentido, la reforma de 1933, como la de 1931, pudo intensificar lo que se conocía como política de masas y hasta reforzar medianamente el papel de los partidos organizados, capaces de enrolar en sus filas a amplios sectores de la opinión y de ganar en circunscripciones tan grandes como la provincia. Pero, contra lo que en un principio pudo suponerse, no significó la desaparición inmediata de las viejas tradiciones políticas individualistas que habían estado plenamente vigentes en nuestra historia electoral y que los partidos republicanos de izquierda y de centro acabaron reproduciendo con mayor intensidad.

Esta percepción parece confirmarse teniendo en cuenta las dos únicas experiencias prácticas con esa ley, las que tuvieron lugar con los comicios generales de noviembre de 1933 y febrero de 1936. En los primeros, la negativa del PSOE a pactar una alianza de carácter nacional con los republicanos de izquierda y el definitivo desplazamiento de los radicales hacia el centro pulverizó cualquier posibilidad de resucitar la conjunción republicano-socialista que se había presentado relativamente cohesionada durante las elecciones a Cortes constituyentes de junio de 1931. Como hemos adelantado, en 1933 existía la idea de que, con la nueva ley electoral, en las provincias donde concurriesen varias candidaturas sería muy difícil que algún candidato sobrepasase el tope del cuarenta por ciento y que la contienda decisiva para la atribución de los escaños sería la segunda vuelta. El hecho de que las derechas sí lograran anudar un pacto nacional no distorsionaba demasiado esas predicciones, pues con los resultados de 1931 y los de abril de 1933 en la mano, no era previsible que sobrepasaran por sí mismas tal tope, sobre todo en las circunscripciones más pobladas (por tanto con más escaños) del país. De ahí que, antes de pactar alianzas, republicanos y socialistas quisieran ponderar fuerzas para, en caso de segunda vuelta, exigirse mutuamente un número de puestos proporcional al número de votos que aportaban en una futura candidatura de coalición. El resultado de la definitiva ruptura de la conjunción republicano-socialista y de la reorganización de las derechas fue un altísimo nivel de concurrencia a los comicios. La cifra oficial de proclamados que anunció el Ministerio de la Gobernación el 12 de noviembre de 1933 (el domingo anterior a las votaciones) fue de 2.077 candidatos, aunque el número se redujo a 1.875 cinco días después merced a la retirada de una parte de los que contaban con menores posibilidades de hacerse con los 473 escaños de las primeras Cortes ordinarias de la República.

Las previsiones de republicanos y socialistas erraron al no medir correctamente la amplitud de la marea conservadora. Las candidaturas de derecha y las "antimarxistas" pactadas entre éstas y los radicales, lograron vencer y sobrepasar el tope en cuarenta de las sesenta circunscripciones. Por el contrario la izquierda sólo logró hacer esto en dos 
(Barcelona provincia y Gerona) gracias a la fuerza de ERC, mientras que los socialistas fueron los más votados en varias circunscripciones (las dos de Madrid, Málaga y Murcia, así como en Ceuta y Huelva) pero sin conquistar en ninguna el tope. Los radicales y sus aliados demostraron algo más de vitalidad y consiguieron vencer y pasar ese tope en seis circunscripciones. Esto hizo que de los 377 escaños ya adjudicados en la primera vuelta, las derechas conquistasen $206(54.6 \%)$ con el 47.5 por ciento de los votos, el centro 112 (29.7\%) con sólo el 19.5 por ciento del voto, y la izquierda sólo 59 (15.7\%) amasando el 33.0 por ciento de los sufragios. Si no se hubieran establecido topes y, por tanto, no hubiera existido la segunda vuelta, la distribución hubiera sido algo más equilibrada, pues la izquierda habría sumado 54 escaños más, llegando a los 113, frente a una derecha que se habría adjudicado 32, logrando 238, y al centro que sólo habría ganado nueve más, alcanzando los 121. No obstante, la segunda vuelta dio una nueva oportunidad a derechas, radicales y republicanos moderados de derrotar a la izquierda habilitando candidaturas conjuntas en las circunscripciones donde sobre todo los socialistas habían demostrado mucha fuerza. El triunfo general de las candidaturas de centro o de derecha en las pocas demarcaciones donde se fue a la segunda vuelta hizo que la izquierda sólo pudiese obtener, tras la jornada electoral del 3 de diciembre, 32 escaños más frente a los 61 del centro y la derecha unidos.

La desproporción parecía aún más aguda descendiendo a escala provincial. No cabe duda de que el establecimiento de cupos tan exagerados para las mayorías distorsionó la representación de las distintas fuerzas políticas. En Badajoz, la candidatura conjunta de radicales, republicanos moderados y derechas se había hecho, ya en la primera vuelta, con once de los catorce escaños de esta circunscripción (el 78.6\%) amasando el 50.2 por ciento de los votos. Los socialistas, que habían cosechado en esta provincia el 48.5 por ciento de las papeletas, quedaban con sólo tres escaños (el $21.4 \%$ de la representación). En Barcelona capital ocurrió de igual forma: con un resultado apretadísimo (un $36.4 \%$ de votos para la Lliga y un $36.3 \%$ para la Esquerra) la derecha catalanista se llevó catorce escaños por cinco de ERC. El resto de candidaturas, que sumaron nada menos que un 27.3 por ciento del voto, quedaron sin representación parlamentaria. Al contrario, durante la segunda vuelta, en Madrid capital los socialistas hicieron triunfar a sus trece candidatos con un 49.7 por ciento de los votos, mientras que la candidatura de derechas sólo obtuvo cuatro escaños pese a haber cosechado el 48.2 por ciento de todos los sufragios emitidos. En otras circunscripciones el resultado fue, en general, menos igualado pero la atribución de escaños continuó siendo, en relación con los votos obtenidos, igualmente desproporcionada (Irwin, 1991; Villa García, 2008).

En febrero de 1936 volvieron a repetirse tales distorsiones. La izquierda y un sector del centro (Unión Republicana) aprendieron la lección de lo ocurrido en 1933 y habilitaron una coalición nacional, el Frente Popular, para unir fuerzas y competir con mayores garantías de triunfo frente a sus rivales. Por el contrario, confiados en el considerable volumen de voto obtenido en noviembre de 1933, y enfrentados por el balance de la política posibilista en el segundo bienio (Álvarez Tardío, 2002: 344-348), la CEDA y el resto de las formaciones políticas de derecha no constituyeron una alianza alternativa articulada a nivel nacional y dejaron que los comités provinciales configurasen totalmente las candidaturas según las particularidades de cada circunscripción. El resultado fue que el centro-izquierda se presentó a los comicios con mayor cohesión, mientras que las disensiones entre monárquicos y republicanos moderados impidieron agrupar a todo el 
centro-derecha en algunas provincias, resultando que frente a las que se denominaron "candidaturas antirrevolucionarias" concurrieron también candidatos disidentes aislados que, necesariamente, habrían de restarles votos. En realidad, los efectos de la reforma electoral se hicieron notar mucho más en esta elección que en la anterior. A diferencia de lo ocurrido en octubre y noviembre de 1933, la segunda vuelta perdió importancia en las consideraciones estratégicas de los partidos. Esto hizo que en casi todas las provincias se generalizase una lucha bipolar y, sólo en muy pocas, hubo terceras candidaturas con un volumen de voto parejo a las "frentepopulistas" o a las "antirrevolucionarias". El número oficial de candidatos proclamados, según datos del Ministerio de la Gobernación, descendió a 987 (ABC, 10-II-1936; El Sol, 11-II-1936). En la práctica, el día antes de la elección concurrieron un total de 942, aproximadamente la mitad que en 1933. No obstante, como resulta bien conocido, lo que en ningún caso se rebajó fue el número de partidos políticos que participaron en los comicios.

El efecto, natural e inevitable, de la menor presencia de aspirantes fue que la primera vuelta, la celebrada el 16 de febrero de 1936, fue la que marcó el resultado final de la elección. Sólo en cinco circunscripciones hubo de repetirse ésta (Álava, Castellón, Guipúzcoa, Soria y Vizcaya provincia). Como es conocido, en una contienda muy igualada en cuanto a número de votos, triunfó la coalición de izquierdas al acumular mayor número de actas de diputado que sus rivales. La clave principal fue que el Frente Popular ganó, y por tanto se llevó las jugosas primas a la mayoría, en las grandes circunscripciones, aquéllas donde las candidaturas contendían por un número de escaños mayor. A efectos de la ley electoral, no importaba que la victoria general de la izquierda hubiera sido apretada. Lo esencial, nada más, fue el hecho de posicionar a sus candidatos en primer lugar. Así, en Jaén el Frente Popular consiguió el 50.5 por ciento de los votos pero diez de los trece escaños en juego (el 76.9\%), mientras que con el 49.2 por ciento de los sufragios, las derechas sólo pudieron copar las tres actas restantes. De forma muy similar, en Valencia provincia el Frente Popular acaparó también las diez actas de las mayorías al quedar en primer lugar con el 45.4 por ciento de los votos (esto es, ni tan siquiera con la mayoría absoluta de los votos), mientras que la Derecha Regional Valenciana hubo de conformarse con los tres de las minorías cosechando el 44.1 por ciento de los sufragios. Esta desproporción se notó incluso en las circunscripciones donde el resultado no fue tan apretado. Así, en Madrid capital, la izquierda amasó un 54.3 por ciento del total de los votos emitidos pero esto le permitió conquistar trece actas de diputado de las diecisiete en juego. La derecha, con el 45.7 por ciento restante, hubo de conformarse con cuatro. En Oviedo, una diferencia algo más apretada (53.0\% para el Frente Popular, $47.0 \%$ para el Frente Antirrevolucionario) también otorgó trece escaños a la izquierda por cuatro de la derecha. De modo que la ley electoral concedió el triunfo a la candidatura, en este caso la del Frente Popular, que supo llevarse la victoria en las circunscripciones estratégicas, es decir, donde la desproporción entre mayorías y minorías era mayor.

He aquí por qué con una cifra global de votos tan igualada, incluso con el hecho evidente de que la izquierda no logró la mayoría absoluta de los votos en 1936, el Frente Popular se colocase ya por delante en los recuentos la noche del 16 de febrero y su victoria (incuestionable, pero en absoluto aplastante) se fuese confirmando con el transcurso de los días. En efecto, la coalición nacional de las izquierdas obtuvo el apoyo de 4.434.111 electores, frente a los 4.402.811 de las distintas candidaturas antirrevolucionarias "oficiales" que las derechas (en algunas circunscripciones incluyendo candidatos 
"portelistas", radicales o de la derecha republicana) articularon en las distintas circunscripciones. Los candidatos disidentes de izquierda que compitieron con el Frente Popular fueron poquísimos y apenas si reunieron cuatro mil votos (3.930 exactamente) en todo el país. No obstante, los candidatos del centro que se presentaron al margen de las coaliciones antirrevolucionarias sumaron 468.242 papeletas, los del PNV reunieron 141.495, y las candidaturas disidentes de derecha 127.369 (de las que Falange sólo obtuvo 10.147 votos de media en todo el país, pues la mayoría correspondían a candidatos agrarios y monárquicos). Cierto que el centro y las derechas demostraban sumar más apoyos, pero no supieron convertirlos en una victoria sencillamente porque en bastantes circunscripciones no consiguieron habilitar candidaturas cohesionadas por completo. Sin duda, los conservadores subestimaron la fuerza de la izquierda y, además, mostraron poco interés por atraer a los electores más centristas creyendo probablemente que los sucesos de 1934 bastarían para que éstos apoyasen, sin más, sus listas. La ley electoral les perjudicó por sí misma pero también las derechas no ponderaron correctamente, a la hora de confeccionar sus candidaturas, el volumen de voto que podía arrastrar el Frente Popular. Por ello, con un 46.3 por ciento de los sufragios emitidos, la izquierda obtuvo ya en primera vuelta 259 escaños (el 57.2\%), mientras que sus rivales, con un porcentaje de voto superior (el 53.7\%) sólo pudieron llevarse 194 escaños. De éstos, la derecha (con un 46.0\% más otro $1.3 \%$ de las candidaturas disidentes), acaparó 162 actas (el 35.8\%), mientras que el centro apenas si cosechó el 4.9 por ciento y la mayoría abrumadora de sus 30 diputados (entre "portelistas", progresistas, radicales, liberales demócratas y "mauristas", un 6.6\% del total de actas atribuidas en primera vuelta) los consiguió en coalición con la derecha. Por último, los nacionalistas vascos sólo obtuvieron, el 16 de febrero, los dos escaños de las minorías en la circunscripción de Vizcaya capital, pues en las tres restantes de la región ninguna candidatura alcanzó el tope del cuarenta por ciento.

Estos resultados han sido elaborados a partir del Legajo 141 del Archivo del Congreso de los Diputados. Por ser los datos oficiales remitidos por las Juntas Provinciales del Censo, resultan mucho más ajustados que los de Javier Tusell (Tusell, 1971). Además, como ya reconoció este historiador, su método fue el de contabilizar los sufragios obtenidos por el candidato más votado de cada lista sin tener en cuenta los votos de las candidaturas minoritarias o unipersonales, sistema que perjudicaba al centro y a la derecha que, en 1936, se presentaron a los comicios con niveles menores de cohesión que la izquierda. Ese método, desde luego, fue impuesto por las circunstancias, pues Tusell y su equipo no contaban con los resultados completos. Nosotros hemos preferido, sencillamente, calcular la media aritmética de los votos de cada candidatura (incluidas las unipersonales), método que permite, sin excluir a ningún partido, hacernos una idea mucho más ajustada de la correlación de fuerzas existente. Los datos oficiales presentan una limitación: el expediente de Cuenca no se encuentra disponible y hemos utilizado los que ofrece el Boletín Oficial de la Provincia de Cuenca (21-II-1936) que coinciden con los que aparecen en las credenciales de los diputados electos por esta circunscripción.

La segunda vuelta tuvo, aunque a menor escala dado el reducido número de circunscripciones en que se celebró, los mismos efectos que la de 1933 pero al revés, ahora perjudicando más aún a las derechas. Sin ésta, los partidos conservadores hubieran alcanzado once de los veinte escaños en juego, por cinco del Frente Popular, tres del PNV y uno del centro (concretamente, un republicano conservador). Con la segunda ronda, las 
derechas sólo lograron tres escaños, mientras que la izquierda sumó ocho, los nacionalistas vascos siete, y el centro otros dos.

En definitiva, ambos comicios mostraron de forma fehaciente las consecuencias que para la representación tuvo la reforma electoral de 1933. La agudización del sistema mayoritario respecto al establecido en 1907 daba la impresión de procurar, más que un simple reforzamiento parlamentario del partido (o coalición) triunfante, el drástico recorte de la representación de las formaciones políticas derrotadas. En este sentido, las predicciones de los que se opusieron a la reforma durante su tramitación parlamentaria se ajustaron a lo que ocurriría con posterioridad. No era que el sistema electoral implantado fuese factor decisivo de la Guerra Civil, afirmación que resultaría a todas luces exagerada. Pero sí contribuyó a aumentar la tensión política al primar la reunión de todas las fuerzas políticas españolas en dos bloques y al sobrevalorar la victoria (por tanto, al agravar los efectos de por sí negativos de la derrota) estableciendo cupos de escaños para las mayorías tan desproporcionados como arbitrarios. Claro que, a la altura de junio de 1933, ni los republicanos de izquierda ni los socialistas, reunidos en torno al gabinete Azaña, supieron avistar lo que se les vendría encima en noviembre de ese año, pues habían concebido la reforma como un mecanismo que asegurase, ad aeternum, mayorías parlamentarias de los partidos que consideraban "sostenedores" del régimen, esto es, los que habían participado de la extinta conjunción republicano-socialista. No habría de pasar siquiera un semestre para que el resultado de unas elecciones hundiera tales expectativas. Del mismo modo, las derechas tampoco supieron impulsar su derogación, quizás bajo los efectos embriagadores de la victoria electoral de noviembre de 1933. Eso, y una defectuosa articulación de las alianzas, malograron la enorme movilización que llevaron a cabo entre enero y febrero de 1936, y provocaron una derrota que acontecimientos posteriores hicieron trascendental.

\section{BIBILIOGRAFÍA}

ALCALÁ-ZAMORA, N. (1977): Memorias (Segundo texto de mis memorias), Barcelona, Planeta. ÁlVAREZ TARDÍO, M. (2002): Anticlericalismo y libertad de conciencia, Madrid, Centro de Estudios Políticos y Constitucionales.

- (2003): "El Parlamento de la Segunda República Española (1931-1936)", en VVAA: El Parlamento en la historia, Vitoria, Servicio de Publicaciones del Parlamento Vasco.

AZAÑA, M. (1997): Diarios 1932-1933: los “cuadernos robados”, Barcelona, Crítica.

CAMPBELL, P. (1958): French electoral systems and elections since 1789, Londres, Faber and Faber LTD.

CARO, D. (1997): "Implantación territorial y modernización organizativa del PSOE en la Andalucía de la Segunda República (1931)", en FORNER, S. (Coord.): Democracia, elecciones y modernización en Europa. Siglos XIX y XX, Madrid, Cátedra, pp. 449-464.

DARDÉ, C. (2000): “Avanzar retrocediendo. La reforma electoral española de 1878”, en MALAMUD, C.: Legitimidad, representación y alternancia en España y América Latina: las reformas electorales (1880-1930), Méjico, Fondo de Cultura Económica, pp. 19-40.

- (2003): “La ley de 'sufragio universal' de 1890", en DARDÉ, C: La aceptación del adversario. Política y políticos de la Restauración, 1875-1900, Madrid, Biblioteca Nueva, pp.197-217, Reed.

DE CARRERAS, F. (1983): "Los intentos de reforma electoral durante la Segunda República", Revista de Estudios Políticos, no 31-32, pp. 165-197.

DEL REY, F. (2008): Paisanos en lucha. Exclusión política y violencia en la Segunda República española, Madrid, Biblioteca Nueva. 
DÉROZIER, A. (1965): "Les discussions sur la loi électorale espagnole en 1835 et en 1836: le gouvernement en échec", en Caravelle: cahiers du monde hispanique et luso-brésilien, ${ }^{\circ}$ 4, pp. 179233.

ESTRADA, M. (1999): El significado político de la legislación electoral en la España de Isabel II, Santander, Universidad de Cantabria.

GARRIDO, A. (2000): "La reforma electoral de 1890", en MALAMUD, C: Legitimidad, representación y alternancia en España y América Latina: las reformas electorales (1880-1930), Méjico, Fondo de Cultura Económica, pp. 41-61.

GUNTHER, R., SANI, G. y GOLDIE S. (1986): El sistema de partidos políticos en España. Génesis y evolución, Madrid, CIS.

HUARD, R. (1997): "Las prácticas del sufragio universal en Francia entre 1848 y 1914. Avances pioneros, novedades provisionales, proyectos inacabados", en FORNER, S. (coord.): Democracia, elecciones y modernización en Europa. Siglos XIX y XX, Madrid, Cátedra, pp. 47-71.

JULIÁ, S. (1995): "Sistema de partidos y problemas de consolidación de la democracia", en Política en la Segunda República, Madrid, Marcial Pons, pp. 111-139.

LINZ, J.J. (1979): El sistema de partidos en España, Madrid, Narcea.

MACARRO, J.M. (2000): Socialismo, República y revolución en Andalucía (1931-1936), Sevilla, Universidad.

RÉMOND, R. (1990): "Les réformes électorales en France au XIXe et XXe siècles", en NOIRET, S. (ed.): Political Strategies and Electoral Reforms: Origins of Voting Systems in Europe in the 19th and 20th Centuries, Baden Baden, Nomos, pp. 108-117.

TOMÁS VILLARROYA, J. (1968): El sistema político del Estatuto Real: 1834-1836, Madrid, Instituto de Estudios Políticos.

TUSELL, J. (1970): "Para la sociología política de la España contemporánea: el impacto de la ley de 1907 en el comportamiento electoral", Hispania, no 116, pp. 571-631.

- (1971): Las elecciones del Frente Popular, Madrid, Cuadernos para el Diálogo, Vol. 2.

VARELA, S. (1978): Partidos y parlamento en la Segunda República, Esplugas de Llobregat (Barcelona), Ariel.

VILLA GARCÍA, R. (2008): La modernización política de España. Las elecciones nacionales de 1933, Tesis doctoral de la Universidad Rey Juan Carlos, Madrid.

\section{Breve currículo:}

\section{Roberto Villa García}

Doctor en Historia y Profesor Titular Interino adscrito al Departamento de Ciencias Sociales de la Universidad Rey Juan Carlos (Madrid). Se ha especializado en Historia Política, especialmente en elecciones y vida política durante la transición a la democracia y la Segunda República. Ha dedicado a esos estudios cuatro monografías y media docena de artículos científicos. Ha sido, además, profesor invitado en las Universidades de Wisconsin-Madison (EEUU) y París IV-Sorbonne (Francia). 
